\title{
Mapeo de áreas inundadas utilizando imágenes de radar TanDEM-X en Acapulco de Juárez, Guerrero-México.
}

\section{Mapping of flooded areas in Acapulco de Juárez, Guerrero-México, using TanDEM-X radar images.}

\author{
Pablo Flores-Lorenzo*a ${ }^{* a}$ Norma Dávila-Hernández*b \\ ${ }^{a}$ Posgrado de Geografía, Universidad Nacional Autónoma de México, Ciudad Universitaria, 04510, Coyoacán, CDMX, México \\ ${ }^{b}$ Laboratorio de Ciencia y Tecnología de Información Geográfica, Facultad de Geografía, Universidad Autónoma del Estado de México, México
}

\section{Resumen}

El presente mapa tiene por objetivo evidenciar el uso de imágenes de radar de alta resolución TanDEM-X en la identificación de zonas inundadas asociadas a la tormenta tropical "Manuel" ocurrida entre el 14 y 16 de septiembre de 2013, en el municipio de Acapulco de Juárez. Se empleó un proceso semi-automatizado mediante técnicas de transformación vectorial reversible para segmentar áreas inundadas, con base en características texturales relacionadas con razones de cambio pre y post-evento. Los resultados permitieron identificar tres tipos de textura asociada al comportamiento de respuesta de la señal de radar, que son relacionados con los distintos estados de cambio de los cuerpos de agua. La utilidad de esta cartografía radica en su contribución al mapeo inmediato (rapid mapping) durante un evento de desastre al emplear imágenes de radar que pueden ser procesadas en corto tiempo; sin embargo, los resultados dependen del nivel de adquisición y polarimetría; así como la inmediatez para adquirir la imagen SAR post-evento.

Palabras clave: Imágenes de radar; TanDEM-X; Inundación; Segmentación; Mapeo inmediato

\begin{abstract}
The present map has as an objective to evince the use of TanDEM-X high-resolution radar images to identify flooded zones associated with Hurricane Manuel, which occurred on September 14th -16th 2013, in the municipality of Acapulco de Juarez, Mexico. A semi-automated process was utilized together with reversible vector transformation to segment flooded areas on the basis of textural characteristics related to pre and post-event change ratios. The results allowed the identification of three sorts of textures associated with the behavior of the radar signal response; these textures are related with various states of change of waterbodies. The usefulness of this cartography lies in its contribution to rapid mapping over a disaster event as it utilizes radar images that can be processed in short time; however, results depend on acquisition level and polarimetry, as well as on the immediateness to acquire post-event SAR images.
\end{abstract}

Keywords: Radar images; TanDEM-X; Flooding; Segmentation; Rapid mapping

\section{Introducción}

De acuerdo con el último reporte de la Base de Datos Internacional de Eventos de Emergencia (The International Disaster

\footnotetext{
${ }^{\sqrt{3}}$ (C) P. Flores-Lorenzo and N. Dávila-Hernández. Published by Terra Digitalis.

This is an Open Access article distributed under the terms of the Creative Commons Attribution License (https://creativecommons.org/licenses/by-nc-sa/4.0/), which permits non-commencial sharing of the work and adaptions, provided the original work is properly cited and the new creations are licensed under identical terms.

Correos electrónicos: pflpablo77@gmail.com, nadavilah@uaemex.mx
}

Database, EM-DAT), la ocurrencia de inundaciones es el desastre con mayor recurrencia e impacto en el mundo y, en los últimos 20 años, el aumento en su frecuencia se ha relacionado con las variaciones climáticas experimentadas a nivel mundial. Un ejemplo de esto es la ocurrencia de fenómenos hidrometeorológicos de gran intensidad que vienen acompañados de precipitaciones extraordinarias y que junto con el crecimiento de las ciudades han producido cuantiosas pérdidas humanas y económicas; así, en el periodo de 1995 a 2015, ocurrieron 3,062 inundaciones a nivel mundial que dejaron a su paso 157,000 pérdidas humanas y afectaron a 2.3 billones de personas, mien- 
tras que las pérdidas económicas alcanzaron 662 billones de dólares. Por lo que se puede decir que las inundaciones son uno de los fenómenos naturales con mayor impacto en el sector económico (UNISDR, 2015).

En los últimos 15 años el uso de datos de observación satelital para el estudio de la prevención del riesgo y soporte, ante el manejo de un desastre, se ha incrementado (Voigt et al., 2016), permitiendo de esta forma que el monitoreo constante y la detección espacial a detalle de los fenómenos naturales sean una alternativa eficiente para la fase de respuesta inmediata al desastre. En especial, el uso de imágenes de Radar de Apertura Sintética (Synthetic Aperture Radar, SAR), al operar independientemente de la iluminación solar y tener la capacidad de contrarrestar, en cierto grado, las condiciones atmosféricas adversas, particularmente la presencia de nubosidad resulta una herramienta eficiente para el mapeo inmediato (rapid mapping), ante una situación de emergencia, por la ocurrencia de fenómenos atmosféricos extremos.

En este trabajo se presenta la cartografía de la inundación asociada a la tormenta tropical "Manuel" (ocurrida entre el 14 y 16 de septiembre de 2013 en Acapulco de Juárez, México), mediante el procesamiento de imágenes SAR. Dicha cartografía se derivó de un método semi-automatizado de segmentación para identificar diferencias en la distribución del backscattering, o respuesta de la señal de radar, asociadas a cuerpos de agua y áreas inundadas.

La utilidad de este tipo de cartografía consiste en contribuir al mapeo inmediato durante un evento de desastre mediante el uso de imágenes de radar, además de demostrar cómo estas imágenes son potencialmente útiles para ser usadas en un Sistema de Alerta Temprana.

\section{Antecedentes}

Históricamente, la ciudad de Acapulco ha sido afectada por ciclones tropicales que han impactado a México. En octubre de 1997, el huracán Paulina dejó a su paso 147 pérdidas humanas, 50,000 damnificados y pérdidas económicas por 447.8 millones de dólares (CENAPRED, 2004).

Años más tarde, en septiembre de 2013, dos ciclones tropicales ocurrieron de manera simultánea en México: en el océano Pacífico, la tormenta tropical "Manuel" y el huracán "Ingrid" en el Golfo de México, afectando el $77 \%$ del territorio nacional. Entre el 14 y 16 de septiembre se presentaron precipitaciones intensas que alcanzaron $885 \mathrm{~mm}$ (Figura 1), esta cifra representa el $80 \%$ de la media anual de precipitación (CONAGUA, 2013). En Acapulco de Juárez, la tormenta tropical "Manuel" ocasionó inundaciones por el desbordamiento de los ríos Papagayo y La Sabana, así como en el complejo turístico denominado Acapulco Diamante y la zona del aeropuerto internacional, las cuales causaron 24 pérdidas humanas y daños por 387 millones de dólares en carreteras, puentes, aproximadamente 13,000 viviendas afectadas, además de 40,000 turistas varados en la zona hotelera (IMPLAN, 2015).

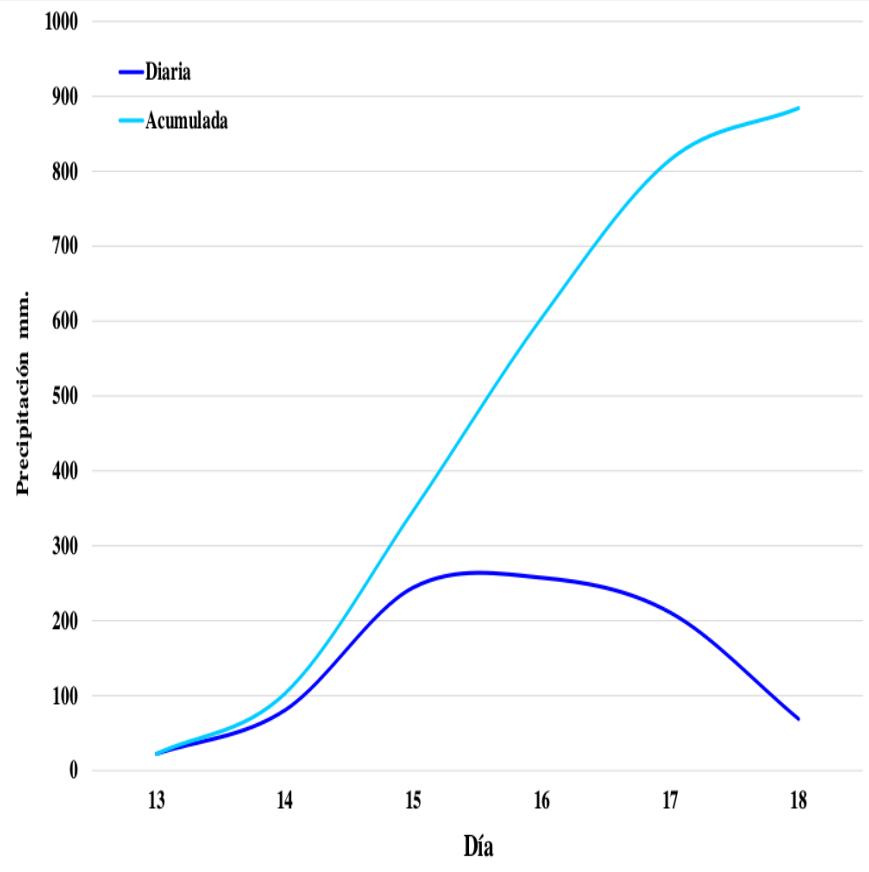

Figura 1. Precipitación diaria y acumulada en milímetros de la cuenca del río La Sabana, del 13 al 18 de septiembre de 2013.

Figure 1. Daily precipitation and accumulation in millimeters for the basin of river La Sabana, September 13 - 18, 2013.

\section{Materiales y Métodos}

Las técnicas utilizadas para el monitoreo de áreas inundadas que usan imágenes de radar, generalmente se basan en el análisis de imágenes cercanas al pre y post-evento de inundación. En particular, la segmentación de cuerpos de agua abiertos es un proceso digital práctico de aplicar, pues generan un retorno homogéneo bajo de la señal al radar, gracias a su reflexión especular. Este retorno aumenta en intensidad al interactuar con estructuras verticales o semi-verticales, características de ambientes urbanos o de cobertura vegetal (Pulvirenti et al., 2010).

Diversos trabajos se enfocan en la generación de algoritmos semi-automatizados para delinear inundaciones mayores, mediante la detección de cambios basados en el comportamiento estadístico de la retrodispersión de la señal (Barstch et al., 2008; O'Grady et al., 2011; Hostache et al., 2012; Clement et al., 2017). Del mismo modo, otros métodos integran el análisis textural y la identificación de límites en la frecuencia del histograma para la segmentación de las áreas inundadas (Pradhan et al., 2014; Nazir et al., 2015; Mason et al., 2016). En este mismo sentido, existen trabajos que muestran la generación de modelos que, además de las técnicas ya mencionadas, tratan de analizar los mecanismos de doble rebote de señal de radar (double bounce scattering mechanism) que se presentan en usos de suelo urbano y forestal inundados (Pulvirenti et al., 2010; Mason et al., 2014; Schumann et al., 2016; Plank et al., 2017).

Para la generación de la cartografía se emplearon dos imá- 
genes TanDEM-X, adquiridas en modo ScanSAR-SC (del 6 de septiembre de 2013) y StripMap-SM (del 28 de septiembre de 2013), con el nivel de procesamiento de un producto de imagen realzado (EEC-Enhanced Ellipsoid Corrected), polarización HH, en órbita ascendente (Tabla 1).

Es importante mencionar que las imágenes fueron programadas como parte del sistema de alerta del Servicio de Información Agroalimentaria y Pesquera (SIAP), que al momento del evento de inundación era el único organismo con telemetría de radar disponible en el país (Figura 2). Las imágenes de tipo EEC, tienen una resolución espacial reducida y cuentan con la corrección geométrica de más alto nivel de los productos TanDEM-X.

En primera instancia, se realizó la normalización del coeficiente de retrodispersión (backscattering) $\sigma_{0}(\mathrm{~dB})$, dado por la forma (Hanssen, 2001): $\sigma_{0}(d B)=10 \cdot \log _{10}($ coeficiente de energía $)$

donde:

coeficiente de energía $=\frac{\text { energía recibida por el sensor }}{\text { energía reflejada de forma isotrópica }}$

Para ambas imágenes se aplicó el filtro de Lee en el espacio de la imagen para la modelación del ruido (speckle), debido a que permite valores de salida cercanos a la media local en áreas uniformes y una estimación de la varianza asociada al ruido global de la imagen, seleccionando de manera interactiva una región homogénea de la misma donde el ruido es implícito, considerando un kernel 5x5 (Lira, 2010). Para generar el mapa de inundación se utilizaron técnicas de transformación digital con el objetivo de caracterizar el comportamiento del backscattering asociado a las áreas inundadas. En primer lugar, se llevó
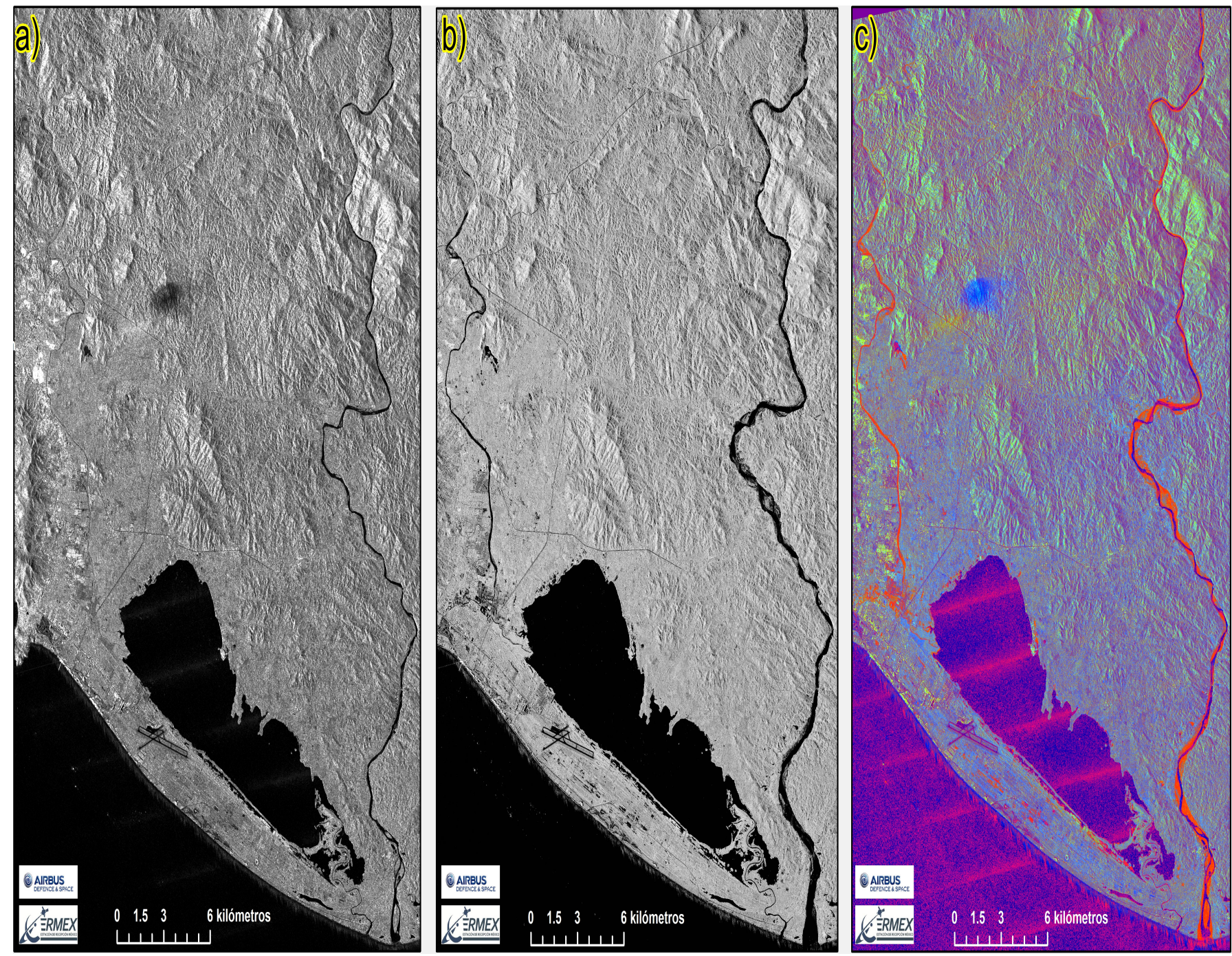

Figura 2. a) Imagen TanDEM-X del 6 de septiembre de 2013 (pre-evento); b) Imagen de radar TanDEM-X del 28 de septiembre de 2013 (post-evento); c) Resultado del tratamiento de la imagen del 28 de septiembre de 2013 (post-evento) TanDEM-X.

Figure 2. a) TanDEM-X image of September 6, 2013 (pre-event); b) TanDEM-X image of September 28, 2013 (post-event); c) Processed TanDEM-X image of September 28, 2013 (post-event). 
Tabla 1: Características de las imágenes TanDEM-X

Table 1: TanDEM-X images characteristics

\begin{tabular}{|c|c|c|c|c|c|c|c|c|c|}
\hline Imagen & Sensor & $\begin{array}{c}\text { Modo de } \\
\text { adquisición }\end{array}$ & Polarización & $\begin{array}{l}\text { Ángulo de } \\
\text { incidencia }\end{array}$ & Órbita & $\begin{array}{c}\text { Dirección } \\
\text { de la órbita }\end{array}$ & $\begin{array}{c}\text { Resolución } \\
\text { espacial (m) }\end{array}$ & $\begin{array}{c}\text { Nivel de } \\
\text { procesamiento }\end{array}$ & Fecha de adquisición \\
\hline $\begin{array}{l}\text { Pre- } \\
\text { evento }\end{array}$ & TanDEM-X & $\begin{array}{c}\text { ScanSAR- } \\
\text { SC }\end{array}$ & $\mathrm{HH}$ & 39.55 & 136 & Ascendente & 8.25 & $\begin{array}{c}\text { Enhanced } \\
\text { Ellipsoid } \\
\text { Corrected } \\
\text { (EEC) }\end{array}$ & 6-septiembre-2013 \\
\hline $\begin{array}{l}\text { Post- } \\
\text { evento }\end{array}$ & TanDEM-X & $\begin{array}{l}\text { StripMap- } \\
\text { SM }\end{array}$ & $\mathrm{HH}$ & 41.50 & 136 & Ascendente & 3 & $\begin{array}{c}\text { Enhanced } \\
\text { Ellipsoid } \\
\text { Corrected } \\
\text { (EEC) }\end{array}$ & 28-septiembre-2013 \\
\hline
\end{tabular}

a cabo la normalización de las imágenes pre y post-evento para estimar la razón de cambio, además de aplicar la transformada vectorial reversible de Componentes Principales (Hotelling, 1933), con la intención de obtener valores asociados a la máxima varianza. Como resultado de la aplicación de esta transformada, se obtuvieron dos imágenes de salida. El proceso de segmentación de las áreas inundadas y cuerpos de agua se realizó con base en las propiedades texturales de las imágenes, esto derivado de la aplicación de una transformada vectorial, que se convirtió en una nueva banda asociada al cambio en el terreno propiciado por la inundación, lo que demostró cambios precisos en las frecuencias de los pixeles que brindaron información importante acerca del estado de la inundación. Posteriormente, se realizó una composición en falso color para facilitar una combinación lineal de los datos correspondientes a distintos estados de inundación y su asociación a diversos usos del suelo y medio natural. Para segmentar las zonas inundadas se utilizó un algoritmo basado en objetos, donde la selección del grupo de pixeles que los compone toma en cuenta atributos espectrales, espaciales y texturales, a diferencia de los clasificadores tradicionales, donde sólo se considera la media de las características espectrales (Jin y Paswaters, 2007).

\section{Resultados y discusión}

En la Lámina 1 se presentan en conjunto las áreas inundadas identificadas con imágenes de radar, sin embargo, se identificaron tres tipos de textura asociada al comportamiento de respuesta de la señal de radar, que a su vez muestra los distintos estados de cambio de los cuerpos de agua. De esta forma, el proceso de segmentación de áreas consideró tanto el lecho de los ríos Papagayo y La Sabana, como el canal de desbordamiento de estos dentro de una reflexión tipo especular, la cual en términos de segmentación de objetos es la más fácil de identificar debido a una disminución uniforme en la intensidad de respuesta de la señal; vinculado así a una textura fina que es característica de las superficies de agua en calma. En segundo lugar, se identificaron las reflexiones de tipo doble rebote (double bounce scattering mechanism) asociadas a cuerpos de agua en zonas urbanas, en su mayoría de tipo habitacional, ubicadas a lo largo del borde oeste del río La Sabana; de manera similar, el proceso de segmentación incluyó los campos de cultivo inundados en los alrededores y los ubicados en la barra que limita a la Laguna de Tres Palos. Finalmente, el tercer grupo fue relacionado con zonas de manglar inundadas, que en su mayoría correspondían al desbordamiento de la Laguna de Tres Palos y Barra Vieja, y que presentaron, de igual manera, una respuesta de doble rebote del sensor. Es importante señalar que la imagen de radar posterior al fenómeno natural fue adquirida 12 días después del evento máximo, por lo que hubo un descenso en el nivel de la inundación y de algunos cuerpos de agua; por lo que los ubicados principalmente al norte de la Laguna no pudieron ser detectados mediante esta técnica.

Con la finalidad de realizar un comparativo de los resultados, se consultaron otras fuentes oficiales que se añadieron como coberturas al mapa final. Entonces, se presenta el área identificada por CONAGUA (2013), que reporta haber utilizado fotografías áreas, dos imágenes ópticas de satélite adquiridas el 18 y 23 de septiembre, al igual que un modelo hidráulico bidimensional y su proceso de validación en campo; todo esto la convierte en la fuente que reporta una mayor extensión de la inundación. Mejía-Estrada (2014) realizó una modelación numérica de la inundación, con base en datos Lidar del INEGI (2007), de muy alta resolución y con datos de información meteorológica, hidrológica e hidrodinámica, identificando el sector habitacional, concentrado en el borde oeste del río La Sabana, como el área más afectada, seguida por la barra al sur de la Laguna; esto verificado por medio de mediciones en campo y con imagen del satélite RapidEye del 18 de septiembre. Por su parte, el 3 de octubre de 2013, la Secretaría de Desarrollo Urbano y Obras Públicas (SEDUOP, 2015) generó un análisis de las causas de las inundaciones en la zona turística Acapulco Diamante, en donde se incluyó un diagnóstico y un reporte a nivel colonia de las afectaciones causadas por la tormenta tropical, siendo la caracterización de las zonas inundadas la de mayor semejanza con los resultados cartográficos obtenidos en este trabajo.

Las discrepancias en la distribución espacial de las áreas inundadas propuestas en el presente trabajo con respecto a las estimaciones realizadas por CONAGUA (2013) y Mejía-Estrada (2014), se pueden explicar por el uso de diferentes metodologías e insumos empleados. CONAGUA (2013) generó su carto- 
Mapa de áreas inundadas en Acapulco de Juárez, Gro., mediante técnicas SAR

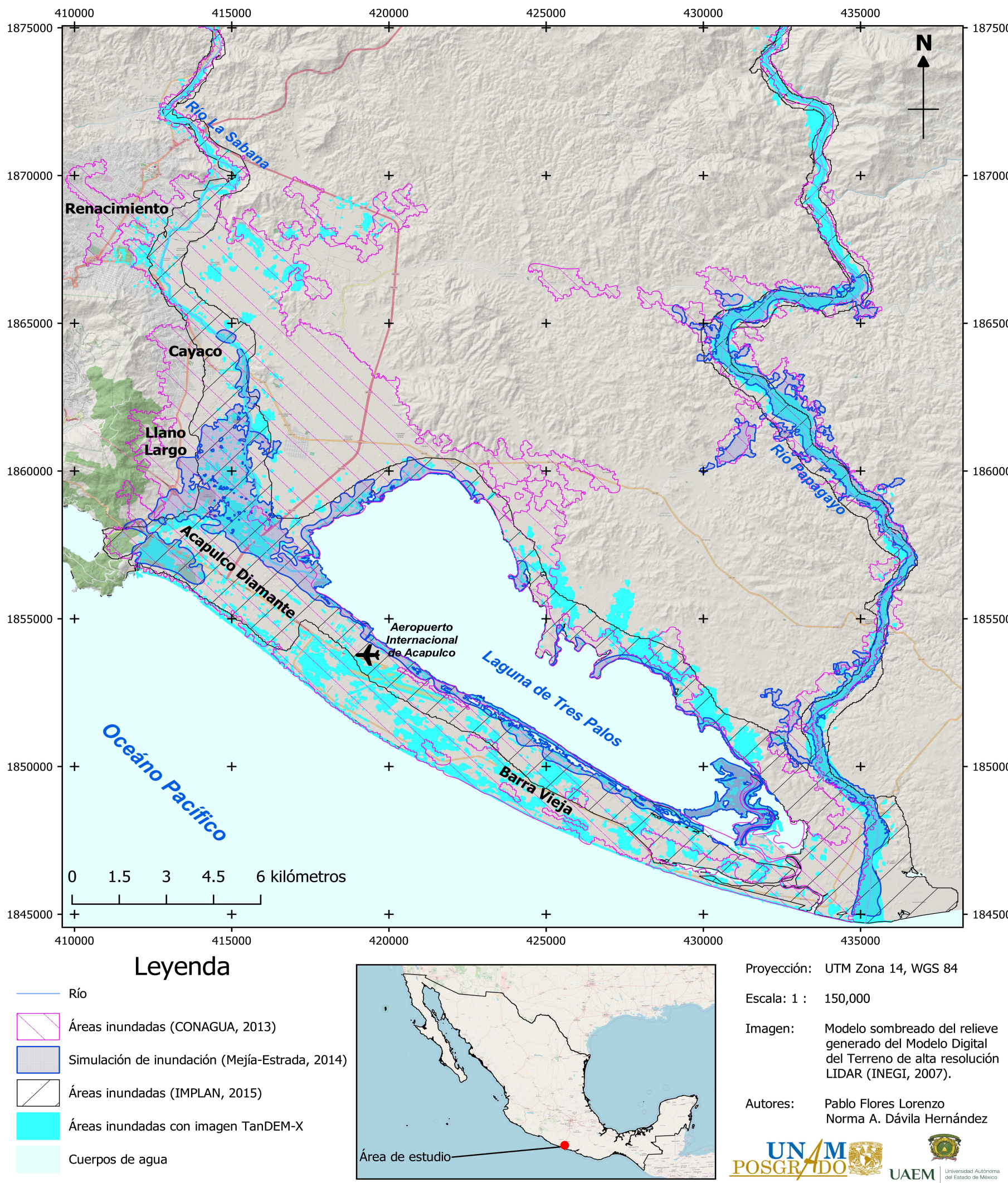

Lámina 1: Mapa de las áreas inundadas en Acapulco de Juárez, Guerrero, por la tormenta tropical "Manuel", septiembre de 2013. Plate 1: Map of flooded areas in Acapulco de Juárez, Guerrero, by tropical storm "Manuel", September 2013. 
grafía a partir de la fusión de resultados entre el modelo hidráulico bidimensional y la estimación de cambio empleando imágenes ópticas. Al contar con información más cercana al evento, delimitó una mayor área; además, el modelo hidráulico simuló áreas que aparecen como no inundadas en los resultados derivados del procesamiento de imágenes satelitales. Por su parte, los resultados reportados por Mejía-Estrada (2014) se obtuvieron a partir de la reconstrucción del evento, empleando datos de precipitación y características del terreno por medio de datos Lidar de 2007, por lo que las discrepancias pueden deberse a que después de 6 años la infraestructura urbana pudo haber experimentado cambios espaciales considerables. Adicionalmente, la simulación sólo se calculó a lo largo de los ríos La Sabana y Papagayo, dejando fuera de este análisis áreas como la Zona Diamante, el aeropuerto internacional de Acapulco, Barra Vieja y las inmediaciones de la Laguna de Tres Palos, las cuales también presentaron afectaciones por inundación, dando como resultado un polígono con una menor área afectada por inundación.

\section{Conclusiones}

La importancia de este trabajo radica en el uso de técnicas semi-automatizadas y de procesamiento práctico para generar una cartografía eficaz e inmediata, dependiendo de la disponibilidad de imágenes, que puedan ser utilizadas en una situación de emergencia o en el manejo de la planeación de alerta temprana. En el caso de las imágenes ópticas, estas pueden demorar en su adquisición o bien, su utilidad puede resultar limitada debido a la presencia de nubosidad, mientras que una imagen SAR puede ser adquirida prácticamente bajo cualquier condición atmosférica, en un menor tiempo, con la calidad necesaria para ser procesada y obtener la información de manera oportuna ante el impacto inmediato de este tipo de desastres. No obstante, la calidad de los resultados puede depender en gran medida de la prontitud para adquirir la imagen post-evento, así como su polarimetría y nivel de procesamiento, ya que resulta indispensable su adquisición en niveles primarios, con la finalidad de realizar las calibraciones adecuadas para la correcta aplicación de este tipo de técnicas semi-automatizadas. La técnica presentada en este trabajo para la generación de cartografía por inundación podría ser mejorada al integrar otros procesos semi-automatizados, como la modelación del comportamiento estadístico de las zonas de cambio o la identificación de límites en la frecuencia de los histogramas, con la finalidad de obtener una mayor exactitud en la segmentación y clasificación de los estados de una inundación.

\section{Software}

El tratamiento digital de las imágenes de radar y ópticas se realizó con el software ENVI 5.1, SNAP 5.0, ERDAS 2015 y Sentinel 1 Toolbox con los que se aplicaron los módulos de corrección geométrica, radiométrica y de segmentación. La edición y diseño final del mapa se llevó a cabo en el sistema de información geográfica QGIS 3.0 Girona, considerando los resultados de otros autores (CONAGUA, 2013; Mejía-Estrada, 2014; SEDUOP, 2015) para cotejar los resultados obtenidos.

\section{Agradecimientos}

Los autores agradecen a los revisores anónimos por brindar su tiempo y esfuerzo, así como sus invaluables aportaciones para la mejora de este trabajo. De igual forma, los autores externan su agradecimiento al SIAP y a la Estación de Recepción México (ERMEX) por la disposición y autorización del uso de las imágenes TanDEM-X en este estudio.

\section{Referencias}

Bartsch, A., Pathe, C., Wagner, W., Scipal, K., 2008. Detection of Permanent Open Water Surfaces in Central Siberia with ENVISAT ASAR Wide Swath Data with Special Emphasis on the Estimation of Methane Fluxes from Tundra Wetlands. Hydrology Research 39(2), 89-100, doi: 10.2166/nh.2008.041

Centro Nacional de Prevención de Desastres (CENAPRED), 2004. Inundaciones. Serie Fascículos. Secretaría de Gobernación, México, 56 pp.

Clement, M. A., Kilsby, C. G., Moore, P., 2017. Multi-temporal synthetic aperture radar flood mapping using change detection. Journal of Flood Risk Management, 1-17, doi:10.1111/jfr3.12303.

Comisión Nacional del Agua (CONAGUA), 2013. Datos de precipitación, gasto hidráulico y áreas afectadas en el municipio de Acapulco, Gro. Secretaria de Medio Ambiente y Recursos Naturales, México, 10 pp.

Hanssen, R. F., 2001. Radar Interferometry: Data Interpretation and Error Analysis. Springer Science \& Business Media, 298 pp.

Hostache, R., Matgen, P., Wagner, W., 2012. Change detection approaches for flood extent mapping: How to select the most adequate reference image from online archives? International Journal of Applied Earth Observation and Geoinformation 19, 205-213, doi:10.1016/j.jag.2012.05.003.

Hotelling, H., 1933. Analysis of a complex of statistical variables into principal components. Journal of Educational Psychology 24, 417-441 and 498-520.

Instituto Municipal de Planeación (IMPLAN), 2015. Programa de colaboración con organismos internacionales. Secretaria de Planeación y Desarrollo Económico, Acapulco, Guerrero, México, 28 pp.

Instituto Nacional de Estadística y Geografía (INEGI), 2007. Modelo Digital de Elevación de alta resolución Lidar, bloque conformado por los modelos en formato de nube de puntos con clave: E14C57A4, E14C57B3, E14C57B4, E14C57D2, E14C57E1, E14C57E2, E14C57E3, E14C57E4, E14C57F1, E14C57F3, E14C57F4, E14C67C1, E14C67C2, E14C57A3, E15A75, E15A76, E15A85, E15A86.

Jin, X., Paswaters, S., 2007. A fuzzy rule base system for object-based feature extraction and classification. Signal Processing, Sensor Fusion, and Target Recognition XVI (I. Kadar, editor). Proceedings of SPIE 6567, pp. 65671H1-65671H12, doi:10.1117/12.720063.

Lira, J., 2010. Tratamiento Digital de Imágenes Multiespectrales. Universidad Nacional Autónoma de México, México, 632 pp.

Mason, D. C., Giustarini, L., Garcia-Pintado, J., Cloke, H. L., 2014. Detection of flooded urban areas in high resolution Synthetic Aperture Radar images using double scattering. International Journal of Applied Earth Observation and Geoinformation 28, 150-159, doi: 10.1016/j.jag.2013.12.002.

Mason, D. C., Trigg, M., Garcia-Pintado, J., Cloke, H. L., Neal, J. C., Bates, P. D., 2016. Improving the TanDEM-X Digital Elevation Model for flood modelling using flood extents from Synthetic Aperture Radar images. Remote Sensing of Environment 173, 15-28, doi:10.1016/j.rse.2015.11.018.

Mejía-Estrada, P. I., 2014. Caracterización del evento hidrometeorológico extremo en Acapulco, Guerrero, en septiembre de 2013. Tesis de maestría, Posgrado Ingeniería Civil-Hidráulica. Universidad Nacional Autónoma de México, 76 pp. 
Nazir, F., Riaz, M. M., Ghafoor, A., Arif, F., 2015. Brief Communication: Contrast-stretching- and histogram-smoothness-based synthetic aperture radar image enhancement for flood map generation. Natural Hazards and Earth System Sciences 15, 273-276, doi:10.5194/nhess-15-273-2015.

O'Grady, D., Leblanc, M., Gillieson, D., 2011. Use of ENVISAT ASAR Global Monitoring Mode to complement optical data in the mapping of rapid broadscale flooding in Pakistan. Hydrology and Earth System Sciences 15, 34753494, doi:10.5194/hess-15-3475-2011.

Plank, S., Jüssi, M., Martinis, S., Twele, A., 2017. Mapping of flooded vegetation by means of polarimetric Sentinel-1 and ALOS-2/PALSAR2 imagery. International Journal of Remote Sensing 38 (13), 3831-3850, doi:10.1080/01431161.2017.1306143.

Pradhan, B., Hagemann, U., Tehrany, M. S., Prechtel, N., 2014. An easy to use ArcMap based texture analysis program for extraction of flooded areas from TerraSAR-X satellite image. Computers \& Geosciences 63, 34-43, doi:10.1016/j.cageo.2013.10.011.

Pulvirenti, L., Pierdicca, N., Chini, M., 2010. Analysis of Cosmo-SkyMed observations of the 2008 flood in Myanmar. Italian Journal of Remote Sensing / Rivista Italiana di Telerilevamento 42 (1), 79-90, doi:10.5721/ItJRS20104217.

Schumann, G. J.-P., Frye, S., Wells, G., Adler, R., Brakenridge, R., Bolten, J., Murray, J., Slayback, D., Policelli, F., Kirschbaum, D., Wu, H., Cappelaere,

P., Howard, T., Flamig, Z., Clark, R., Stough, T., Chini, M., Matgen, P. Green, D., Jones, B., 2016. Unlocking the full potential of Earth observation during the 2015 Texas flood disaster. Water Resources Research 52, 3288 3293, doi:10.1002/2015WR018428.

Secretaría de Desarrollo Urbano y Obras Públicas (SEDUOP), 2015. Análisis y causas de las inundaciones en la zona Diamante, tormenta "Manuel", septiembre 2013. Gobierno del Estado de Guerrero, México, 38 pp.

Voigt, S., Giulio-Tonolo, F., Lyons, J., Kucera, J., Jones, B., Schneiderhan, T., Platzeck, G., Kaku, K., Hazarika, M., Czaran, L., Li, S., Pedersen, W., Kadiri James, G., Proy, C., Macharia, D., Bequignon, J., Guha-Sapir, D., 2016. Global trends in satellite-based emergency mapping. Science 353 (6296), 247-252, doi:10.1126/science.aad8728.

Wallemacq, P., Guha-Sapir, D., McClean, D., CRED, UNISDR, 2015. The Human Cost of Weather Related Disasters 1995-2015. Doi: 10.13140/RG.2.2.17677.33769.

This article accompanies the following material:

HTML:

Static map:

Interactive map:
DOI: 10.22201/igg.terradigitalis.2018.1.36.85

DOI: 10.22201/igg.terradigitalis.2018.1.36.96

DOI: 10.22201/igg.terradigitalis.2018.1.36.97 